\title{
Linx
}

Revue des linguistes de l'université Paris X Nanterre

$58 \mid 2008$

Aspects de comme

\section{Comme en diachronie : bilan}

Mélanie Morinière et Thomas Verjans

\section{OpenEdition}

\section{Journals}

Édition électronique

URL : http://journals.openedition.org/linx/337

DOI : 10.4000/linx.337

ISSN : 2118-9692

\section{Éditeur}

Presses universitaires de Paris Nanterre

\section{Édition imprimée}

Date de publication : 1 juin 2008

Pagination : 141-162

ISSN : 0246-8743

Référence électronique

Mélanie Morinière et Thomas Verjans, «Comme en diachronie : bilan », Linx [En ligne], 58 | 2008, mis en ligne le 16 février 2011, consulté le 20 avril 2019. URL : http://journals.openedition.org/linx/337 ; DOI : 10.4000/linx.337

Département de Sciences du langage, Université Paris Ouest 


\title{
Comme en diachronie : bilan
}

\author{
Mélanie Morinière E Thomas Verjans \\ ENS-LSH (Lyon) - ICAR \& Paris Sorbonne - STIH (EA 4089)
}

\section{Introduction}

Les études synchroniques portant sur le morphème comme se sont récemment multipliées, notamment sous l'influence du développement des études liées aux proformes indéfinies. En revanche, la dimension diachronique est demeurée quelque peu négligée, malgré l'engouement suscité par les interrogations touchant au problème du changement linguistique. Pour autant, la question de l'évolution et de la filiation des diverses valeurs de comme a toujours fait l'objet d'une attention particulière et de nombreuses études l'évoquent de façon plus ou moins explicite. Les travaux menés sur comme peuvent se répartir en quatre ensembles, lesquels organiseront les subdivisions de cette présentation : d'abord les travaux qui concernent l'époque romane, c'est-à-dire le passage du quomodo latin au comme ${ }^{1}$ de l'ancien français; puis ceux, assurément plus nombreux, qui portent sur la valeur subordonnante (ou conjonctive) assumée par ce morphème ; ceux qui concernent ses autres valeurs, c'est-à-dire non subordonnantes ${ }^{2}$;

\footnotetext{
${ }^{1}$ Pour simplifier, on désignera le morphème par la forme comme quel que soit l'état de langue étudié, mais cette forme n'apparaît que tardivement: en français médiéval, on trouve de très nombreuses orthographes, les plus fréquentes étant com et come (c'est pourquoi les linguistes traitant de l'ancien français utilisent souvent la forme $\operatorname{com}(e))$

2 Nous avons conscience des limites d'une terminologie aussi traditionnelle que celle qui oppose emplois subordonnants et emplois non subordonnants. Elle nous semble cependant légitime en ce sens que, n'appartenant pour ainsi dire à personne, cette terminologie ne risque pas de véritablement
} 
et enfin, les travaux à visée plus synthétique, qui s'attachent à ordonner l'ensemble des valeurs dans le cadre d'une perspective évolutive plus générale. Nous comprenons donc ici le terme "diachronie» au sens large, puisque nous intégrons aussi dans cette présentation les études portant sur le morphème dans tel ou tel état ancien de langue, c'est-à-dire, à strictement parler, des études synchroniques. Toutefois, lorsque cela sera possible, nous essaierons de conserver la perspective diachronique, c'est-à-dire évolutive, en rendant compte de la filiation des valeurs postulée par les auteurs évoqués. Une limite doit néanmoins être posée : dans cette synthèse ne seront considérés que les ouvrages ou articles spécifiquement consacrés à comme ou, à tout le moins, comportant une analyse exclusive et détaillée. Sont donc écartés les ouvrages de référence, tels les grammaires usuelles et les dictionnaires, dont les analyses sont en outre le plus souvent reprises par les travaux dont nous rendons compte.

\section{Du latin aux langues romanes}

Deux études complémentaires concernent comme en roman : celle de J. Herman (1963) et celle de R. de Dardel (1983).

Dans son ouvrage sur La Formation du système roman des conjonctions de subordination (1963), J. Herman répartit l'étude de quomodo / com(e) d'une part dans l'évolution du système latin, d'autre part dans le système des premiers textes romans. En conséquence, cette étude, sans envisager la situation strictement romane, n'en offre pas moins des informations extrêmement précieuses sur l'avant et l'après. Il souligne ainsi, comme le rappelait aussi P. Imbs avant lui (1956: 118-119), que le comme français, et plus généralement la forme romane apparentée, trouve son origine dans le composé latin quomodo, et non pas dans la particule cum comme cela a pu être parfois soutenu ${ }^{3}$. Et si l'on peut admettre, comme le fait J. Herman,

que la fausse identification des particules romanes dont nous nous occupons, avec la conjonction latine cum n'est pas restée sans influence sur l'évolution des particules romanes dans la langue écrite [HERMAN $1963: 173-174]^{4}$,

il n'en reste pas moins qu'

il est certain cependant que cette influence n'a fait que hâter la réalisation de possibilités qui étaient incluses d'emblée dans les fonctions de quomodo et de ses continuateurs. [HERMAN 1963 : 173-174]

Le terme quomodo, véritable étymon de comme, est un adverbe de manière, formé de deux ablatifs, quo $(<q u i)$ et modo ( $<$ modus). Aussi bien contient-il dès l'origine le

trahir les choix des auteurs concernés par chacune de ces manchettes non plus que d'orienter par trop un compte-rendu qui, sans être neutre, se veut tout de même aussi objectif que possible.

${ }^{3}$ On pourra consulter, au sujet de cette confusion, les travaux suivants : Imbs (1956: 118-119); Herman (1963 : 173-174) et Pierrard (2001 : 294-295).

${ }^{4}$ Herman ajoute : «Il est évident que les gens qui savaient du latin identifiaient cum, com français avec cum latin; on en a une preuve formelle dans le passage suivant de l'Orthographia Gallica (éd. J. Stürzinger : XI-XII, H 26-27 Et altrefoithe escriveretz c vel q indifferenter come cuer ou qoer. Et altrefoithe solonc le Latyn ut qi, cum, qe. » [1963: 170, note 3] 
" sème ", au sens propre, de manière, que renforcerait encore, s'il en était besoin, le fait qu'il s'agisse de deux formes à l'ablatif ${ }^{5}$. C'est donc comme adverbe de manière que le terme quomodo s'inscrit dans le latin classique où il connaît à l'origine deux grandes séries d'emplois. Il intervient d'abord - et avant tout - dans le domaine interrogatif, qu'il s'agisse d'interrogation directe ou d'interrogation indirecte ${ }^{6}$. Il intervient également comme relatif, au sens de «de la manière dont, ainsi que ». Dans les deux cas, il apparait bien que le terme s'unifie autour de ce sème de /manière/.

Sans davantage entrer dans les détails d'un sujet qui excèderait par trop les limites de notre propos, on gagnera néanmoins à rappeler que, dès la période latine, quomodo connait une évolution qui lui confèrera la plupart des valeurs que $\operatorname{com}(e)$ retrouvera par la suite. Un certain nombre d'entre elles sont liées à l'instauration progressive d'une substitution à $u t:$ «quomodo remplaçait de plus en plus souvent $u t$ dans plusieurs de ses fonctions » $(1963: 45)^{7}$. C'est notamment la valeur comparative qu'intègre quomodo, laquelle sera, pour certains auteurs, à l'origine de toutes les valeurs d'emploi suivantes. Ainsi, toujours selon J. Herman,

Meyer-Lubke (...) a sans doute raison en supposant que l'emploi temporel de notre conjonction s'explique à partir de son emploi comparatif: le parallélisme, la ressemblance de deux actions ou de deux états implique souvent leur rapprochement dans le temps, ce qui, dans la psychologie des sujets parlants, facilite des glissements de la fonction comparative vers la fonction temporelle et cela d'autant plus que l'identité partielle déjà existant entre les valeurs de quomodo et celles de $u t$ donnait à des glissements de cet ordre une « justification » analogique. [HERMAN $1963: 58$ ]

À cette valeur temporelle, très largement attestée, J. Herman ajoute également une valeur causale, possiblement dérivée de la valeur temporelle ${ }^{8}$, et, beaucoup plus rare, une valeur finale (ibid. : 59). Enfin, la dernière valeur d'emploi évoquée par J. Herman, est la valeur conjonctive complétive après les «verba sentiendi et dicendi » (ibid. : 44), c'est-à-dire les verbes de perception et de propos. Cette valeur serait plus tardive (à partir du IVe s.) et résulterait d'une grammaticalisation de l'emploi interrogatif indirect, grammaticalisation par ailleurs favorisée par le fait que

la langue vulgaire n'a jamais été conséquente dans l'emploi du subjonctif en interrogation indirecte $(. .$.$) , ce qui contribuait à rendre la limite incertaine entre$

\footnotetext{
${ }^{5}$ Rappelons, en effet, que la manière est, en latin classique, l'un des sens dérivés de l'ablatif proprement dit (cf. Ernout \& Thomas 1953 : $\int 108$ ). L'on peut dès à présent remarquer que parmi ces valeurs figurait également la « conformité » (Ibidem).

${ }^{6}$ À ce propos, Ernout \& Thomas (1953: \ 181c et \318) observent que, dans cet emploi, quomodo est « usuel» au sens de « comment».

${ }^{7}$ En quoi J. Herman retrouve l'idée couramment évoquée selon laquelle se manifestait, dans les emplois de quomodo, une «tendance à éliminer les constructions indicatives de $u t$ » [Ernout \& Thomas 1953: \352] ainsi que l'installation d'une concurrence entre les deux formes, notamment dans l'expression de la comparaison où quomodo est alors présenté comme un «supplétif fréquent de $u t$ de comparaison » [Imbs : $1956: 119]$.

${ }^{8}$ Ce que tendrait à confirmer aujourd'hui les études les plus récentes sur les « chaînes » conceptuelles en jeu au cours de processus de grammaticalisation: voir, par exemple, H. Bat-Zeev Shyldkrot (1995 : 149).
} 
interrogation indirecte et complétive simple, d'autant plus que, dans ces dernières, le subjonctif et l'indicatif alternaient également sans régularité rigoureuse (...). [HERMAN $1963: 45]^{9}$

Quoi qu'il en soit de l'acquisition de ces dernières valeurs, comme connaît donc déjà, à cette époque, l'ensemble de ses valeurs de base.

Étudiant ensuite «les descendants romans de quomodo» (1963: 166-174), J. Herman montre que « dans le cas de cette conjonction, le problème de la transition entre la structure latine et les structures romanes se pose donc presque uniquement sur le plan des fonctions » (1963: 166), c'est-à-dire de ses valeurs d'emplois. Or, le point qu'il nous semble intéressant d'évoquer ici tient aux premières attestations de valeurs dans certaines langues romanes. Si elles connaissent toutes une valeur comparative généralement présente dès les premiers textes, l'on peut remarquer, en revanche, que les valeurs 'secondaires' se génèrent différemment. Ainsi, à l'inverse de l'ancien français, qui voit la valeur temporelle attestée dès les premiers textes et la valeur causale plus tardivement ${ }^{10}$, le vieil espagnol et le vieux portugais connaissent un cheminement inverse : la valeur causale y est en quelque sorte première, et la valeur temporelle n'y apparait que plus tard (1963: 167-168). Cela introduit un certain flottement dans la filiation des valeurs, du moins telle que l'expose J. Herman. Selon lui, en effet, la valeur temporelle aussi bien que la valeur causale peuvent désormais être toutes deux issues de la valeur comparative, ce qui s'accorderait mal avec l'hypothèse avancée précédemment, originant la valeur causale de quomodo dans sa valeur temporelle.

À cela s'ajoutent les emplois en corrélation, brièvement évoqués pour le latin (1963 : 98), mais un peu plus détaillés pour les langues romanes (ibid. 248-253). Pour ces dernières, J. Herman distingue trois types en fonction des catégories grammaticales des termes mis en jeu :

1- Type «adverbe ou pronom + quomodo», la plus fréquente étant formée à partir « d'un continuateur roman de l'adverbe sic et de come, com etc. comparatif ; plus rarement, on rencontre aussi comme corrélatif de come etc. comparatif un des pronoms tantum ou talem » $(1963: 248)$;

2- Type «préposition + quomodo», «locutions [qui] se rencontrent dans les langues romanes de la Péninsule ibérique et dans le Sarde » (1963: 251);

3- Type « préposition + pronom + quomodo», limité au catalan (1963 : 253).

Le terme quomodo se répand ainsi dans l'ensemble des langues romanes, où il est largement attesté, et participe le plus souvent au système primaire des conjonctions de subordination. Le roman connait donc en quomodo un terme potentiellement pourvu de l'ensemble de ces valeurs, même si celles-ci n'apparaissent pas toutes également dans chacune des langues qui lui succèderont. Or, c’est à étudier précisément cette période

\footnotetext{
${ }^{9}$ Notons que, sur ce point, une opposition quelque peu implicite oppose J. Herman à Ernout \& Thomas, pour lesquels l'ensemble des valeurs d'emploi acquises par quomodo s'explique essentiellement par le principe d'une substitution à ut (1953: §352).

${ }^{10}$ Voir notamment Bertin (1997 : 121-124).
} 
transitoire que s'attache R. de Dardel dans son Esquisse structurale des subordonnants conjonctionnels en roman commun (1983), en rappelant tout d'abord que

le roman commun est une abstraction. Nous voulons dire par là que le roman commun est une langue qui n'existe qu'en vertu de postulats fondés sur une série d'extrapolations à partir des parlers romans. [DARDEL 1983 : 38]

Mais il n'en propose pas moins une étude fondée sur une stratification de cette période en une succession de cinq synchronies, principalement distinguées à partir d'un critère géographique. Dès lors, les recherches et les reconstructions postulées lui ont permis d'établir la répartition suivante de l'évolution des emplois de quomodo:

QUOMODO a pour fonctions primaires l'introduction des comparatives d'égalité (3.1./4) et des complétives modales 'comment' (12.2./1); de ces fonctions paraissent issues, dans des aires assez étendues mais contenues dans celles des fonctions primaires, l'emploi de QUOMODO comme subordonnant de temporelles de simultanéité (9.1.2./2), de complétives subjectives et objectives $(12.3 / 2)$, de comparatives hypothétiques d'égalité (3.3.1./1), de causales (1./4), de finales (2./2) et de comparatives proportionnelles (3.4./3). [DARDEL 1983 : 65]

Le détail de ces évolutions retrouve globalement les hypothèses traditionnelles à ceci près, d'une part, qu'il résout en quelque sorte par l'alternative le dilemme manifesté dans l'analyse de J. Herman, en posant que

en français comme causal peut être théoriquement issu soit de QUOMODO comparatif soit de QUOMODO temporel. [DARDEL 1983 : 28] ;

et, d'autre part, que les différentes synchronies postulées offrent une hypothèse explicative aux divergences d'attestations entre les différentes langues romanes. Ainsi l'hypothèse de la séparation précoce du sarde se justifie-t-elle négativement par la présence, partout ailleurs,

de fonctions que (...) QUOMODO (...) n'avai (..)t pas encore en roman commun moyen, à savoir (...) les fonctions causale (1./4), finale (2./2), comparative hypothétique d'égalité (3.3.1./1) et superlative (4./2) (...). [DARDEL 1983 : 52]

De la même façon, concernant l'emploi des tours corrélatifs :

Un peu plus récents (roman commun tardif B), parce qu'absents à la fois du sarde et du roumain, (est) l'emploi (...) de SI ${ }^{1}$-QUOMODO dans les identifiantes (8./3) et les temporelles $(9.1 .2 . / 7)$ (...). Dans cette synchronie apparaissent aussi (...) quelques locutions subordonnantes (...) à radical QUOMODO (3.1./9). Plus récent encore (roman commun tardif $\mathrm{C}$ ), selon le critère spatial (est) l'emploi de QUOMODO dans les proportionnelles (3.4./3). [DARDEL 1983 : 53 ]

Enfin, la diversité d'emplois manifestée par les différentes synchronies du roman s'explique également par la notion de syncrétisme. Ainsi dans le cas de quomodo:

Les syncrétismes ont dû affecter en roman commun des particules originairement différenciées, mais, dans la plupart des cas, proches par la forme phonique et susceptibles de se joindre par évolution phonétique ou morphologique, comme dans (...) QUO / QUOD / QUOMODO (...). [DARDEL 1983 : 57 ] 
Par ailleurs, R. de Dardel postule une coexistence, au moins à la fin de la période classique, des futures formes romanes et des anciennes formes latines, ce qui peut expliquer le chevauchement des différentes valeurs au cours du latin vulgaire (1983: 54-55) et, par conséquent, certains des glissements sémantiques à l'origine de ces valeurs.

Dès le latin et les premières strates romanes, quomodo est donc pourvu d'un sémantisme comparatif qui évolue vers d'autres valeurs circonstancielles : temps, cause et finalité. L'ensemble de ces valeurs sera attesté, à un moment ou à un autre dans l'histoire du français, ensemble auquel il faudra cependant ajouter les valeurs non subordonnantes, notamment adverbiales et, parfois même prépositionnelles, le cas de l'emploi relatif, au sens où l'entend Pierrard (1998), étant le plus généralement inclus sous la notion plus large de subordonnants. Les deux sections suivantes sont consacrées aux travaux amorçant plus spécifiquement les différentes étapes de l'évolution de comme en français, la section II. étant centrée sur les emplois subordonnants, et la section III. sur les emplois non subordonnants.

\section{Emplois subordonnants : Prédication 1 (adv.) comme Prédication 2}

Les emplois subordonnants de comme sont ceux qui, par des biais divers, ont le plus largement retenu l'attention des linguistes. La question majeure ne concerne cependant pas tant l'étude de chacun des emplois pour lui-même, mais la filiation des valeurs propres à chacun d'eux. C'est en ce sens également, et pas seulement de façon chronologique, qu'ils prolongent les travaux recensés sous le point précédent.

\section{1. P. Imbs (1956) : la valeur temporelle de la structure Prédication 1 (adv.) comme Prédication 2}

Dans Les propositions subordonnées temporelles en ancien français (1956), au chapitre consacré à comme, intitulé Temps et manière : «com(e)» et ses composés, P. Imbs s'intéresse aux valeurs circonstancielles de la proposition introduite par comme ou un composé de comme, essentiellement à sa valeur temporelle. L'ouvrage présente une étude principalement synchronique de l'ancien français (IX ${ }^{\mathrm{e}}$-XIII ${ }^{\mathrm{e}} \mathrm{s}$.), mais elle conduit l'auteur à des remarques diachroniques sur l'origine du système de l'ancien français et certaines évolutions après cette période.

Le fonctionnement et la valeur de comme «temporel » sont comparés à ceux de l'autre principal morphème de coïncidence temporelle (simultanéité), quant, qui a fait l'objet du chapitre précédent. Il en ressort d'abord que de même que quant, comme fonctionne seul ou, plus souvent, dans des structures analytiques (les structures « corrélatives » de J. Herman). P. Imbs note que les formations analytiques étaient très fréquentes en ancien français pour réaliser la jonction, complexe, entre deux propositions, et il détaille, comme J. Herman, le fonctionnement des structures avec comme, les différents adverbes que l'on rencontre, etc. Cette comparaison entre comme et quant lui permet en outre d'affiner la question des liens entre les valeurs circonstancielles de comme. Il montre d'abord que les différences entre comme et quant, par exemple l'affinité de comme avec l'adverbe si, mettent en évidence le fait que la valeur temporelle de comme n'est qu'une interprétation en contexte de sa valeur première de «manière d'être/ 
circonstance générale », si étant lui aussi un adverbe de manière devenu adverbe de « circonstance générale ». D’autre part, si la locution «temporelle» si (...) comme a la même forme que la locution exprimant une comparaison d'égalité, cependant la rareté de la locution aussi comme à valeur temporelle, locution exclusivement comparative, montre, selon P. Imbs, que la valeur temporelle n'est pas dérivée de la valeur de comparaison d'égalité, comme l'a écrit W. Meyer-Lübke, repris par J. Herman et de R. de Dardel (voir section I.), mais que toutes deux proviennent de la valeur de manière.

Si dans la plupart des cas comme n'est pas synonyme de quant, dans certaines phrases cependant il semble avoir une valeur strictement temporelle. Pour P. Imbs, cela n'est pas étonnant puisque

le Moyen Age proprement dit a moins connu le travail de différenciation sémantique des synonymes, auquel procéderont le XIIIe, XIVe, puis le XVIIe et le XVIIIe siècles; il ne professe pas encore cette conception principalement logique, suivant laquelle, dans la langue idéale, chaque idée s'exprimerait par un seul mot et chaque mot traduirait une seule idée. [IMBS 1956 : 154]

D'autre part, cet emploi fréquent d'un comme temporel proche de quant (l'adverbe de reprise y est souvent un adverbe strictement temporel comme lors ou dunc) dans certains textes très anciens ou textes de traducteurs et auteurs savants, semble être influencé par l'analogie avec la conjonction latine temporelle cum. L'auteur note aussi que jusqu'en français classique, comme et ses composés, tout comme quant, peuvent être suivis de tous les temps narratifs (passé simple, présent historique, futur, etc.). Mais on peut remarquer dès l'ancien français le début de la différenciation entre comme temporel et quand par la spécialisation de comme dans l'introduction d'une action passée (exprimée par un verbe à l'imparfait) au cours de laquelle surgit un événement nouveau (rendu par un verbe à un temps narratif), valeur que ne peut exprimer quant/quand:

Si com dedens leur nef entroient

Un biau jovencial venir voient. (Image du Monde, cité par P. Imbs, p 137)

"Comme ils entraient dans leur navire, ils voient venir un beau jeune homme »

P. Imbs consacre quelques pages à la fin du chapitre aux autres emplois des subordonnées en comme. Il propose une triple origine au comme causal du français moderne, très rare en ancien français : le latinisme comme suivi du subjonctif, calqué sur le latin cum + subjonctif ; un comme qui n'est qu'une interprétation en contexte d'une circonstance temporelle concomitante; une variante du comme de comparaison, comme... aussi. Comme complétif, lui, serait dérivé de l'interrogatif de manière. Quant à comme final, il souligne que les grammaires n'en citent qu'un exemple, extrait de la Cantilène de Sainte Eulalie, dans lequel il propose de voir un calque du latin quomodo + subjonctif « pour que de cette manière... », où quomodo a une valeur de manière, le sens final venant du mode subjonctif de la relative. 


\section{2. P. Jonas (1971): le fonctionnement des structures comparatives en ancien français.}

L'ouvrage de P. Jonas (1971), Les systèmes comparatifs à deux termes en ancien français est également une étude essentiellement synchronique de l'ancien français $\left(\mathrm{IX}^{\mathrm{e}}\right.$ $\mathrm{XIII}^{\mathrm{s}}$.), basée sur un large corpus d'exemples. Consacré aux structures comparatives, il est complémentaire de l'ouvrage de P. Imbs pour l'étude du fonctionnement de comme puisqu'il approfondit la question des emplois comparatifs du morphème ${ }^{11}$. L'auteur met en évidence cinq structures formelles distinctes exprimant un rapport comparatif entre deux termes. Dans quatre de ces structures (A1-A4), les deux termes sont liés par un morphème "articulant», dans la cinquième (B) ils sont articulés seulement par la mélodie :

(A1) $1^{\text {er }}$ terme - articulant $-2^{\mathrm{e}}$ terme (avec un articulant simple com(e) ou que, ou composé si com(e), si que, ensi com(e), etc.)

(A2) $1^{\text {er }}$ terme + marque - articulant $-2^{\mathrm{e}}$ terme : plus / tans / moins / autre / autresi / si / etc. (marque) riche que / de/com(e) (articulant))

(A3) $1^{\mathrm{er}}$ terme + marque - articulant $-2^{\mathrm{e}}$ terme + marque (plus...com(e)/ que / quant plus...etc.)

(Le diable à propos des hommes)

Plus les fait de mal faire con plus ont d'abundance. (Poème moral, cité par Jonas, p 320)

«Il leur fait faire d'autant plus de mal qu'ils ont de richesses. »

(A4) Articulant $-1^{\mathrm{er}}$ terme + marque $-2^{\mathrm{e}}$ terme + marque $(\operatorname{com}(e) /$ que/quant plus....plus...etc.)

Con plus esmuet (on) la merde, e ele plus put (Proverbes, cité par Buridant, 2000, p 651)

«Plus on remue la merde, plus elle pue.» plus... etc.)

(B) $1^{\mathrm{er}}$ terme + marque, sans mélodie conclusive $-2^{\mathrm{e}}$ terme + marque $($ Plus...,

Son corpus d'exemples permet à P. Jonas d'associer ces cinq structures formelles à trois types sémantiques de comparaison ou opérations cognitives :

- (A1) exprime une «conformité de nature» (entre deux entités) ou «une conformité (d'un fait) à une circonstance » (1971 : 80)

- (A2) exprime une confrontation associée la « commensuration » (comparaison de deux mesures : supériorité, infériorité ou égalité) ou aux caractéristiques (différence ou identité).

- $\quad$ (A3), (A4) et (B) expriment une variation proportionnelle.

\footnotetext{
${ }^{11}$ Le chapitre «La Comparaison» de la Grammaire nouvelle de l'ancien français de Cl. Buridant (2000) reprend quasiment telles quelles les analyses de cet ouvrage.
} 
Comme est utilisé dans les quatre premières structures (A1-A4). Il présente donc différents fonctionnements syntaxiques en ancien français : on le trouve seul, «articulant synthétique », ou comme second élément d'un «articulant analytique » (locution) (1971: 83) dans la structure $\mathrm{A} 1$, ou alors associé à un ou plusieurs adverbes (" marques ») dans un système corrélatif (A2, A3, A4). Mais certains des morphèmes qui constituent le premier élément de la locution (si, autresi, aussi) ayant la même forme que les marques d'égalité du système corrélatif, et la locution n'étant pas toujours soudée, P. Jonas admet qu'il est parfois difficile de décider si l'on a affaire à la locution (exprimant une conformité/analogie) ou au système corrélatif (exprimant une égalité de degrés) (1971: 45).

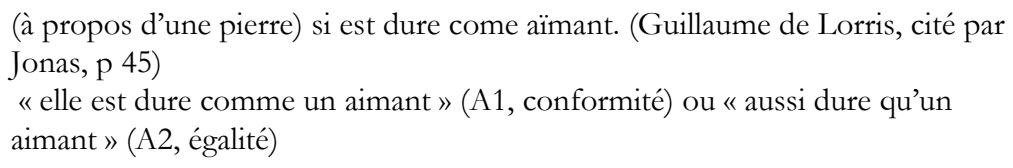

P. Jonas s'intéresse aussi à la distribution entre com(e) et que dans les systèmes comparatifs (1971: 197 sq). En ancien français, com(e) apparaît dans les contextes exprimant une "concordance » : seul ou en locution, il exprime une conformité, dans les structures corrélatives, il est appelé par les adverbes d'égalité si/aussi/autant/etc. (identité de degrés) et d'identité tel/autretel/etc. ; que, lui, apparaît en général avec des marques exprimant une disparité (plus, moins, autre, etc.). Sur la question du remplacement de com(e) par le morphème que, vide de matière notionnelle, dans les locutions composées (fr. mod. ainsi que) et les systèmes corrélatifs (fr. mod. aussi/autant/tel que), l'auteur montre que celui-ci a commencé dès l'ancien français, d'abord dans les contextes où il n'y a pas confrontation entre deux substances différentes, mais conformité à une circonstance, ou bien quand cette confrontation de deux substances est niée ou altérée (contextes non thétiques, etc.) (1971 : 324 sq).

P. Jonas met en outre au jour les tendances de distribution en synchronie entre l'articulant simple comme et les locutions composées du type si com(e) (1971: $81 \mathrm{sq}$ ), d'une part, et entre les différents adverbes (formes en autre-/au-vs si/tant/ensi/tel, si vs ensi, etc.), d'autre part, ainsi que l'évolution de ces morphèmes en diachronie ${ }^{12}$. Dans la seconde partie de l'ouvrage, il étudie les facteurs contextuels qui permettent à ces structures comparatives de créer des « effets de sens », comme le haut degré, ou des « faits d'expressivité ».

\section{3. Autres valeurs circonstancielles de Prédication 1 comme Prédication 2}

L'étude des valeurs circonstancielles de la structure Prédication 1 comme Prédication 2 et la question de leur filiation, question soulevée à maintes reprises dans les ouvrages présentés dans les sections précédentes, a été poursuivie par plusieurs travaux plus récents, sous deux angles distincts mais complémentaires. H. Bat-Zeev Shyldkrot (1989, 1995) d'abord, a entrepris, par le biais de plusieurs articles, d'étudier l'évolution de comme au travers du prisme de la grammaticalisation ; O. Soutet (1992) et

12 Le travail sur la distribution des différents adverbes s'inspire de H. Kjellman (1924) " Autresiaussi-ainsi. Étude de syntaxe historique », Studier i modern språvetenskap, IX : 147 sqq. 
A. Bertin (1997) ont, quant à eux, envisagé comme par le biais de certaines des valeurs sémantiques attachées à son emploi subordonnant, respectivement la concession et la cause ; enfin, A. Kuyumcuyan (2006 et 2007) a poursuivi cette entreprise dans une perspective plus pragmatique.

Prenant acte de la difficulté de distinguer les différentes valeurs sémantiques des propositions subordonnées adverbiales, notamment en raison de la polysémie de leurs marqueurs, H. Bat-Zeev Shyldkrot envisage dans chacun des deux articles les phénomènes généraux à l'œuvre dans l'évolution des conjonctions de subordination, et plus spécifiquement la question de la grammaticalisation.

Dans le premier cas (1989), elle étudie les conjonctions pourvues d'une valeur temporelle-causale, parmi lesquelles figure comme. À l'instar de bien d'autres, comme permet d'illustrer certaines régularités dans l'évolution sémantique des marqueurs de subordination, notamment la prédominance de l'expression d'un rapport de cause non explicite (ou présupposée) et le fait que les conjonctions possédant cette double valeur sémantique «ne traduisent jamais un rapport temporel d'antériorité » (1989: 276). Ce dernier aspect s'explique par le fait que « la fonction prédominante des conjonctions dans l'acte de discours est surtout de nature pragmatique» (ibid.). Elle note en outre, dans le point plus spécifiquement consacré à comme, que si «tout comme pour quand, les différents sens de comme se sont presque tous accumulés en français moderne», celui-ci «ne s'utilise jamais plus aux sens de 'combien' et de 'quel', qui lui étaient propres dans l'ancienne langue, dans les interrogations » (1989: 271).

Poursuivant l'étude de l'évolution sémantique des connecteurs au moyen du modèle de la grammaticalisation, H. Bat-Zeev Shyldkrot s'intéresse dans un second article (1995) aux marqueurs de comparaison, de concession et de condition. Elle «se propose de tracer le parcours d'évolution que ces termes sont susceptibles de suivre et de montrer le lien qui existe entre leur évolution diachronique et leur interprétation synchronique» (1995: 145). Toutefois, si comme est évoqué pour illustrer les brouillages interprétatifs dus à la polysémie des marqueurs, la suite de l'étude concerne davantage les marqueurs à base quantitative (plus que, moins que) et n'expose pas directement la relation entre le sens comparatif de comme et son sens concessif.

Quoique comme soit d'un usage concessif bien moindre que comment et combien, il n'en ignore pas pour autant cette valeur, à tout le moins dans certaines configurations syntaxiques particulières. Celles-ci ont été détaillées par O. Soutet dans son ouvrage, inspiré par la psychomécanique du langage, sur la concession en français. La première d'entre elles relève des «systèmes asymétriques à base adverbo-conjonctive », systèmes qui se mettent en place «à partir du dernier quart du XIV e siècle » et se manifestent sous la forme d'« une syntaxe distributive qui s'articule autour de l'adverbe tant et des conjonctions que ou comme» (1992: 66). Se retrouve ici le problème déjà évoqué par P. Imbs et P. Jonas de la substitution de que à comme, laquelle, dans ce cas et par rapport au corpus utilisé par O. Soutet, semble intervenir au «milieu du XVe siècle avec, comme texte charnière dans notre corpus, Artois» (ibid. : 67).

Par la suite est explicitement posée la question d'un comme à valeur concessive à partir d'occurrences dans lesquelles celui-ci est suivi d'un verbe au subjonctif (ibid. : 145). Pour rare qu'il soit, cet usage n'en apparait pas moins comme un usage savant qui se rencontre notamment dans les traductions de textes de latin. Il n'est pas 
inintéressant, à cet égard, comme le note O. Soutet (ibid.:145, n. 1), de remarquer, outre la graphie cum du texte français, le fait que ce soit également un cum latin qui soit traduit de la sorte. Enfin, cet emploi serait «à rapprocher de l'emploi, sans doute plus fréquent, d'un comme + subjonctif, tout autant démarqué du latin mais de sens causal» (ibid. : 145).

Le dernier emploi à être mentionné par O. Soutet est celui de la locution comme que, «de sens équivalent » aux suites comment que et combien que, «mais d'emploi rarissime » (ibid. : 194). Les occurrences recensées se répartissent cependant de façon tout à fait sporadique entre le XIII et le XVI e siècles, pour finalement céder le pas devant l'usage de comment que, mais, surtout, devant celui de combien que.

Quoique très limité dans le temps aussi bien qu'en nombre d'occurrences, comme connaît donc un usage concessif rare et tardif. De la même façon, l'emploi causal de comme, étudié par A. Bertin (1997: 121-124), est un emploi relativement tardif, très ponctuellement attesté au cours de l'ancien français (essentiellement « dans des textes didactiques d'origine savante» ou dans «des traductions» $(1997: 121)$ ). Cette valeur causale proviendrait, comme le proposait déjà P. Imbs, aussi bien de sa valeur comparative que de sa valeur temporelle :

Malgré sa double filiation temporelle et comparative, come n'affirme sa valeur pleinement causale qu'à partir du XIIIème siècle, dans des textes particuliers et selon des contraintes syntaxiques rigides. D'après P. Imbs cet essor correspond, à l'inverse, à la disparition du quant causal. [BERTIN 1997 : 124]

Les configurations syntaxiques dans lesquelles comme manifeste cette valeur se distinguent d'autant plus nettement de son usage en français contemporain que, si la proposition y est généralement antéposée, elle est également le plus souvent au mode subjonctif. L'expression causale apparaît "très nettement liée à un discours argumentatif, l'enchaînement étant parfois souligné par » doncques, en tête de l'autre membre propositionnel (ibid. : 122), ou encore par le sémantisme des verbes recteurs. Ce n'est cependant qu'à partir du moyen français que se développera de façon plus massive l'usage de cette valeur, aboutissant par la suite à l'emploi que l'on connait aujourd'hui.

L'article d'A. Kuyumcuyan (2006) «Comme et ses valeurs : le point de vue historique (XIV ${ }^{\mathrm{e}-X V I}$ e siècles) » met en avant l'importance de la dimension pragmatique, évoquée dans les remarques d'A. Bertin (1997), pour l'étude de l'évolution des valeurs circonstancielles de comme. Après une section assez générale sur la filiation des valeurs à partir du sens de manière, section reprenant dans une perspective diachronique large les idées de C. Fuchs et P. Le Goffic $\left(1993,2005^{13}\right)$, A. Kuyumcuyan s'intéresse plus particulièrement à l'interprétation causale de la proposition comme $\mathrm{P}$ antéposée, à partir d'un corpus d'occurrences de moyen français. Elle reprend en la développant l'idée de P. Imbs d'un faisceau de facteurs concordants aboutissant à l'attribution d'une valeur causale à comme. Cette valeur pourrait venir d'une part d'une interprétation argumentative de certaines propositions comparatives. L'opération de comparaison est en effet orientée :

${ }^{13}$ Le Goffic, P. Grammaire de la phrase française, Paris, Hachette (1993) ; Fuchs, C. et P. Le Goffic, « La polysémie de comme » in Soutet, O. (ed.) La Polysémie, Paris, PUPS, 2005. 
pour faire saisir au destinataire le contenu propositionnel A de la principale, le comparé, le locuteur le met en rapport avec le contenu B de la subordonnée, le comparant, supposé connu (par le contexte antérieur, des connaissances encyclopédiques ou la doxa) ou plus facilement accessible; le contenu B prépare donc, argumente par anticipation, le contenu A. L'auteure montre par ailleurs comment, dans d'autres occurrences, comme $\mathrm{P}$ exprime une circonstance générale concomitante, valeur dérivée de la valeur de manière, qui est interprétée selon le contexte avec une nuance temporelle (circonstance antérieure) et/ou causale, voire concessive quand les deux événements ne sont pas co-orientés. Enfin, l'interprétation causale de comme $\mathrm{P}$ a dû être confortée par l'influence du cum causal + subjonctif du latin classique, notamment dans la littérature didactique d'inspiration antique très développée à cette époque.

L'originalité de cet article est d'insister sur le fonctionnement textuel de comme $\mathrm{P}$, ce qui permet de mettre en évidence une propriété commune à tous les emplois intégratifs de comme (ou des locutions composées) : sa fonction discursive. Comparatif, temporel/causal ou métadiscursif (si comme est contenu en la Bible), comme « hiérarchise l'information du plus connu au moins connu, du constituant secondaire sur lequel il porte à l'élément principal qu'il amorce et auquel il facilite l'accès » (2006: 125).

S'intéressant toujours au fonctionnement textuel de comme, dans un autre article (2007), A. Kuyumcuyan étudie les tours en comme et dire, comme on dit, comme qui dirait et comme dit/dirait l'autre, marqueurs de modalisation autonymique en français contemporain déjà étudiés par J. Authier-Revuz (1995), grâce auxquels le locuteur commente la forme de son discours, mettant à distance l'expression qu'il utilise en l'attribuant à un autre énonciateur, indéfini. A. Kuyumcuyan retrace grâce à la base Frantext l'histoire de ces tours depuis le XVIe siècle, en proposant une explication au changement de modalité énonciative de ces marqueurs métadiscursifs (comme exprimant la conformité de deux énonciations, de deux dire), qui, marqueurs de discours représenté, signalant l'attribution à un autre locuteur du contenu d'un fragment du discours, sont devenus marqueurs de modalisation autonymique, permettant d'attribuer à un autre énonciateur la forme signifiante du fragment.

L'ensemble des travaux recensés sous ce point permet donc d'aboutir à une connaissance approfondie des différentes valeurs sémantiques assumées par comme en tant qu'élément subordonnant, et de la filiation en diachronie de ces différentes valeurs.

\section{Emplois non subordonnants}

Nombreux sont les emplois dans lesquels comme ne fonctionne pas comme un marqueur de subordination. Ainsi en va-t-il de ses emplois en quelque sorte originaires, dans lesquels il exprime l'interrogation et l'exclamation. Ceux-ci, hors les travaux synthétiques que nous évoquerons plus bas, n’ont cependant jamais véritablement fait l'objet d'études diachroniques, sinon, en quelque sorte, de façon biaisée. Il en va ainsi de l'article de F. Lefeuvre (2003), qui étudie l'histoire du marqueur composé comme quoi. Celui-ci apparait au XVII ${ }^{e}$ siècle en tant qu'adverbe interrogatif de manière, en 
interrogation directe et indirecte (Mais comme quoy ("comment») dompter ce Prothé variable? (Scudéry, cité par F. Lefeuvre 2003 : 456)), peut-être comme une solution à la controverse entre comme et comment qui se disputent l'emploi interrogatif. Comme quoi devient progressivement un introducteur de discours indirect (Ce que je peux faire, c'est mettre comme quoi (*comment) elle a eu la mention TB (énoncé entendu cité par F. Lefeuvre 2003 : 456)). Cette évolution présente selon F. Lefeuvre les caractéristiques d'une grammaticalisation : d'une part, le morphème perd son sens de manière pour acquérir une valeur sémantique plus ténue de marqueur énonciatif; d'autre part, d'un point de vue morpho-syntaxique, il subit une recatégorisation, son fonctionnement interrogatif/ percontatif se modifiant pour se rapprocher de celui d'un complétif, avec toutefois des caractéristiques distributionnelles et une valeur énonciative particulières. Pour F. Lefeuvre, dans la locution comme quoi, comme est un adverbe intégratif.

Un autre emploi non subordonnant de comme a attiré l'attention de plusieurs linguistes : comme dit parfois "prépositionnel », suivi d'un SN, d'un SAdj., ou d'un équivalent. Ces auteurs se sont intéressés en particulier au rapport en synchronie et en diachronie entre le syntagme comme SN comparatif (« échantillant», dans la terminologie de Damourette \& Pichon $^{14}$ ) et le syntagme comme SN/SAdj./etc "attributif» ou «qualifiant». On présentera ici les travaux de V. Väänänen $(1951)^{15}$, de Ph. Ménard (1994), d'E. Tobback (2003) et de M. Morinière (2008a et 2008b).

Un des premiers à étudier spécifiquement le syntagme qualifiant/attributif introduit par comme, et ses liens avec la valeur comparative de comme, est V. Väänänen (1951), dans une monographie diachronique sur les tours du type Il est venu comme ambassadeur ou Il agit en soldat en français, en italien et en espagnol, depuis leurs origines en latin. Il y distingue, parmi les éléments qui «indiqu[ent] l'état, la situation ou la qualité qui revient au sujet ou à l'objet», le "prédicatif» (Il fut nommé ambassadeur), complément indispensable au verbe, de "l'apposition circonstancielle» (Il est venu comme ambassadeur), «sorte de prédicat secondaire et tenant du circonstanciel». Dans cette opposition on reconnait la distinction entre attribut accessoire et attribut essentiel des linguistes français actuels. L'un et l'autre peuvent être réalisés en latin et dans les langues romanes par un SN ou un SAdj. (« apposition ou prédicatif pur(e) et simple»), un syntagme introduit par le morphème employé pour la comparaison (lat. $u t /$ sicut $/$ quasi/tamquam $^{16}$, fr. comme, it. come, esp. como) ou un syntagme introduit par une préposition (fr. en, pour, etc.). L'auteur étudie l'origine de cet emploi de $u t / c o m m e / e t c$. et des prépositions par rapport à leurs valeurs premières, morphème de manière/

\footnotetext{
14 J. Damourette et E. Pichon (1911-1940) Essai de grammaire de la phrase française, Paris, D’Artrey.

${ }^{15}$ V. Väänänen propose une version réduite de cette monographie de 1951 dans le chapitre «Il agit en soldat et tours concurrents » de son ouvrage Recherches et récréations latino-romanes, Napoli : Bibliopolis, pp. 189-216. Il y reprend presque tels quels les chapitres II, III, et IV de la monographie, ceux sur le latin et le français, mais il n'y explique pas sa terminologie, notamment la différence entre « apposition » et « prédicatif».

16 Selon V. Väänänen, l'apposition introduite par les continuateurs de quomodo existant dans toutes les langues romanes, «ce n'est sans doute qu'un accident que quomodo, assez fréquent en bas latin comme conjonction, soit pour ainsi dire inconnu dans la fonction appositionnelle » (1951:22).
} 
comparaison ou préposition spatiale, puis la concurrence, en diachronie, entre les différents procédés (pour le français, attributs directs et attributs introduits par comme, pour, de, à, en tant que, à titre de, etc.).

Sur la question des liens entre comme «qualifiant » et comme comparatif, l'intérêt de cette étude est de montrer que parmi les occurrences dans lesquelles le $\mathrm{SN} / \mathrm{SAdj}$./etc. qui suit comme est interprété comme un prédicat second appliqué au sujet ou à l'objet, il y en a où comme $\mathrm{SN} / \mathrm{SAdj}$. désigne aussi une manière ou une conformité ("comparaison qui implique l'identité » p. 26) comme dans Cunseilez mei cume mi savie bume ("Conseillez moi, vous, (comme) mes hommes sages ») (Chanson de Roland, cité p. 26). Il nous semble même que comme a sa valeur première de conformité dans beaucoup des exemples pourtant cités par l'auteur comme désignant «l'identité sans plus ».

V. Väänänen est par ailleurs l'un des premiers à signaler le développement en français moderne d'un emploi nouveau de comme $\mathrm{N}$ paraphrasable par en fait de $\mathrm{N}$, comme dans Comme (= en fait de) chasseur de casquettes, Tartarin n'avait pas son pareil (Daudet, cité p. 29), emploi qu'il distingue à juste titre de comme $\mathrm{N}$ équivalent de en tant que $\mathrm{N}$ (*En tant que chasseur de casquettes, Tartarin n'avait pas son pareil.) ${ }^{17}$.

Ph. Ménard (1994) s’intéresse également à cet emploi de comme introduisant un syntagme proche d'un attribut dans un article consacré à trois emplois particuliers de comme en ancien français où le morphème, qui n'exprime pas une comparaison prototypique, comparaison entre deux entités distinctes, présente quand même, selon lui, un noyau de sens commun avec comme comparatif : la valeur de conformité. Il montre ainsi, lui aussi, que dans les syntagmes qualifiants de l'ancien français («com(me) faisant référence à une qualité ${ }^{18}$ ) du type Comme droit hoir de France font Pepin coronner ("Ils font couronner Pépin comme héritier légitime du royaume de France ») (Berte aux grans piés, cité par Ménard 1994 : 258) comme exprime une conformité entre le procès auquel participe un individu et une qualité, un statut social ou une fonction possédé par l'individu. Dans la deuxième section, il met l'accent sur un emploi particulier de la proposition introduite par comme, «com(me) dans les formules d'adjuration », qu'il analyse comme une identité de valeur de vérité de deux propositions ('aussi vrai que $\mathrm{P} 1$, aussi vrai P2').

Dites lui, si conme il a chier / M'amor, qu'il ne voist en avant. (Lai de l'Ombre, cité par Ménard 1994 : 260)

« Dites-lui, au nom de l'amour qu'il me porte, qu'il ne poursuive pas sa route »

Dans la troisième section il essaie de distinguer divers emplois souvent confondus de comme $+\mathrm{SN} / \mathrm{SPrep}$. non comparatifs (mais dérivés, selon lui, de la valeur comparative) :

- un tour explicatif (à savoir, c'est-à-dire, par exemple) ou justificatif (comme il convient) dans lequel les deux entités ou procès ont un rapport d'identité ou d'inclusion :

Et sans faille la barate estoit ja conmencie si com des nouviaux cevaliers. (Tristan en Prose, cité par Ménard, 1994 : 266)

\footnotetext{
17 Voir Gautier \& Morinière (ce volume).

18 Dans sa Syntaxe de l'ancien français (1980/94), il nomme cet emploi « fausse comparaison ».
} 
«Et assurément le combat était déjà commencé, à savoir celui des nouveaux chevaliers »

- et le tour approximatif ou atténuatif (environ, presque) :

Tot droit un dyemenche, si com aprés diesner, / Ez vous venu Morant, qui , revient d'Outremer. (Berte aus grans piés, cité par Ménard, 1994 : 265)

«Tout droit un dimanche, peu après dîner, voici Morant qui arrive, de retour d'Outremer»

Ph. Ménard admet toutefois que ces tours sont souvent difficiles à distinguer.

E. Tobback (2003) se pose aussi la même question: le syntagme attribut introduit par comme (elle se limite à la construction à attribut de l'objet (CAO), par exemple considérer, élire $\mathrm{SN}$ comme $\mathrm{SN} / \mathrm{SAdj}$./etc.) est-il dérivé du complément de manière/comparaison ( $\mathrm{CdM}$ ) introduit par comme? Choisissant le cadre des théories de la grammaticalisation, elle utilise les paramètres proposés par C. Lehmann (1995) ${ }^{19}$ pour situer les principales constructions du français moderne faisant intervenir comme sur un continuum de grammaticalité. Ses conclusions vont dans le même sens que les analyses de M. Pierrard (1998, 1999, 2001).

Adv. interr. de manière

$$
\text { CdM verbal CdM non verbal }
$$

$\mathrm{CAO}$ (disparu en fr. mod.)

$$
\text { - gramm } \quad+\text { gramm }
$$

La construction à attribut de l'objet (CAO) étant la plus grammaticalisée juste après le complément de manière $(\mathrm{CdM})$ averbal, la question est: peut-elle être le résultat d'une grammaticalisation du CdM par réanalyse, comme le suggère, par exemple, M. Grevisse dans Le Bon Usage, qui fait dériver Je considère cet homme comme un héros de Je considère cet homme comme je considère un héros (CdM verbal). E. Tobback montre qu'en français moderne en tous cas, la CAO en comme n'est plus analysable comme un $\mathrm{CdM}$, puisque dans de nombreux exemples attestés de CAO on ne peut reconstituer une proposition à partir du segment introduit par comme comme on peut le faire avec un CdM averbal.

Prolongeant les réflexions d'E. Tobback (2003), dans un article sur « l'ellipse dans les constructions en comme en diachronie du français », M. Morinière (2008a) interroge aussi le rapport entre le syntagme comme $\mathrm{X}$ adverbial de manière averbal et le syntagme comme X attributif, mais dans une perspective évolutive, depuis l'ancien français jusqu'au français moderne. Si le recours à l'ellipse a fréquemment cours dans les interprétations de comparatives, tant à des fins interprétatives qu'à des fins métalinguistiques, sa systématisation masque parfois des réalités empiriques distinctes, qu'un retour à la diachronie peut contribuer à mettre en lumière. Dans une première

${ }^{19}$ Lehmann, C. (1995), Thoughts on grammaticalization, München/Newcastle, Lincom Europa. 
partie, M. Morinière propose une étude des segments comparatifs verbaux et averbaux comme $\mathrm{X}$ en ancien français qui conforte l'analyse des segments comparatifs par le recours à la notion d'ellipse pragmatico-sémantique proposée par M. Desmets (2001) ${ }^{20}$ pour le français moderne :

l'interprétation propositionnelle de la séquence non phrastique de droite $\mathrm{X}$ se fait par récupération dans la séquence de gauche non de formes, mais de notions sémantiques, entités et prédicats. [MORINIÈRE 2008a]

M. Morinière remarque cependant quelques différences dans le fonctionnement de l'ellipse entre l'ancien français et le français moderne, notamment l'apparition en français moderne de l'obligation pour comme d'être suivi d'un constituant dit «majeur ».

Dans une seconde partie, elle s'intéresse aux syntagmes dits «attributifs» introduits par comme, et à leur évolution en diachronie large. En français moderne, les syntagmes attributifs de forme comme $+\mathrm{SN}$ sans déterminant, avec $\mathrm{N}$ désignant une fonction ou un statut, comme dans Jean travaille comme maģon, ne peuvent être analysés comme des syntagmes elliptiques avec la même définition de l'ellipse que dans les syntagmes comparatifs: ils ont les propriétés d'un syntagme prépositionnel. En revanche, prolongeant les remarques de Väänänen (1951) sur la «comparaison impliquant l'identité » et de Ménard (1994) sur «com(me) faisant référence à une qualité », M. Morinière montre qu'en ancien français, tous les syntagmes comme SN/SAdj./SPrep de sens attributif (c'est-à-dire dans lesquels le SN ou le SAdj. désigne une propriété attribuée à un objet) peuvent être analysés comme des adverbiaux de manière propositionnels elliptiques. Ayant recours aux concepts des théories de la grammaticalisation, elle propose une hypothèse selon laquelle la construction comme + $\mathrm{N}$ (rôle/fonction) du français moderne serait le résultat de la réinterprétation sémantique et de la réanalyse en diachronie de l'adverbial de manière/comparaison elliptique comme $+\mathrm{N}$ dans certains contextes.

Un autre article de M. Morinière (2008b), toujours en diachronie large, replace l'apparition des syntagmes attributifs introduits par comme dans l'histoire des constructions à attribut de l'objet en général. L'auteure y montre en effet que, alors que l'attribut était toujours construit directement en latin classique, apparaît entre le latin et l'ancien français une nouvelle catégorie grammaticale, les «indices de l'attribut » a, por, puis en, issus de la grammaticalisation des prépositions latines ad, pro et in dans certains contextes. Parallèlement, les syntagmes comme SN/SAdj. adverbiaux de manière à sens attributif (manière ou procès conforme à une qualité d'un objet) sont beaucoup plus fréquents que l'équivalent latin $u t \mathrm{SN} / \mathrm{SAdj}$. Au XIVe siècle, comme apparait même en concurrence avec les « indices 》 $a$ et por avec certains verbes comme réputer, tenir (au sens de "considérer») : comme SN/SA s'éloigne du circonstant de manière, il assume un rôle véritablement attributif, notamment marqué par l'impossibilité de le supprimer. Ce type de constructions va alors gagner d'autres verbes au cours des siècles suivants, éventuellement en fonction de leurs propres modifications sémantiques (passage de la perception à l'opinion pour un verbe comme regarder, par exemple). Dès lors, parallèlement à la généralisation de la construction

\footnotetext{
20 Desmets, M. (2001) Les typages de phrase en HPSG : le cas des phrases en "comme ». Thèse de doctorat. Université Paris X-Nanterre (np).
} 
attributive directe, comme devient l'indice privilégié des constructions indirectes persistantes ou nouvellement créées, l'emploi de $\grave{a}$ en tant qu'indice d'attribut ayant presque disparu et pour n'étant plus productif dans cet emploi.

\section{Travaux à perspective synthétique}

Cette dernière section est consacrée aux travaux qui abordent comme dans une perspective synthétique, ceux de T. Ponchon (1998), V. Wielemans (2005) ainsi qu'un ensemble d'articles, dus à M. Pierrard (1998, 1999, 2001), dont la somme offre également une vision d'ensemble des emplois de comme et de leur évolution.

Dans son étude sur «les emplois de com(e) en français médiéval», T. Ponchon entend

dégager, dans le cadre du français médiéval, à la lumière de la psychomécanique du langage et de la théorie de la subduction, le signifié de puissance de comme, à partir de la polyfonctionnalité qui émerge de ses signifiés d'effets. [PONCHON 1998 : 319]

Il s'agit donc, dans un cadre théorique postulant une unité fondamentale en langue de ce type d'élément linguistique, de mettre au jour le signifié de puissance de comme, susceptible de donner lieu à l'ensemble des valeurs que manifeste sa polysémie discursive. Celles-ci sont ramenées au nombre de six, présentées suivant un mouvement constructeur allant, en termes guillaumiens, d'une « saisie pleinière » à des saisies de plus en plus « subduites » (ibid. : 343):

C 1 : Comme adverbe nominalisateur non comparatif

C 2 : Comme adverbe comparatif

C 3 : Comme adverbe en locution comparative

C 4 : Comme «préposition»

C 5 : Comme conjonction temporelle / causale

C 6: Comme conjonction en locution conjonctive temporelle / causale / concessive

La visée de ce travail est d'autant plus globale qu'il faut entendre ici par «français médiéval» une périodisation incluant l'ancien et le moyen français. En outre, T. Ponchon propose une perspective plus systématique en situant l'ensemble des emplois de comme par rapport à ceux de comment et de combien. Cependant, comme l'on pouvait s'y attendre et comme il est tout à fait légitime pour une étude de ce type, cette visée globale constitue également sa limite, dans la mesure où elle ne permet pas de détailler l'évolution et les éventuelles filiations des différentes valeurs, et en ce sens relève davantage d'une synchronie large que d'une diachronie véritable. Il n'en demeure pas moins qu'elle offre les fondements d'une appréhension systématique, apte à révéler aussi bien le fonctionnement interne de ce terme que les relations qu'il entretient avec les microsystèmes dans lesquels il s'intègre.

La seconde étude à visée synthétique, due à V. Wielemans (2005) et intitulée «L'évolution de comme et comment: le témoignage des grammairiens et des dictionnaires de l'époque ", s'inscrit dans une perspective plus résolument diachronique. Y sont en effet envisagés les différents emplois assumés par comme et comment tels qu'ils étaient 
appréhendés par les contemporains : grammairiens, remarqueurs et auteurs de dictionnaire, mais aussi tels qu'ils sont présentés dans les grammaires modernes. D'autre part, la période couverte par cette étude s'étend de l'ancien français jusqu'à l'aube du français contemporain (XVIII siècle).

Cette étude se fonde d'abord sur une distinction des diverses valeurs d'emploi de chacun des deux termes (2005: $:$ 1.1), regroupées en six catégories pour comme:

Comme 1 : interrogation et exclamation directe et indirecte ;

Comme 2 : Introducteur de subordonnées à valeur circonstancielle ;

Comme 3 : emploi comparatif et valeur « exemplative»;

Comme 4 : comparatif d'égalité, notamment en «locutions conjonctives, telles que aussi comme, tant comme »;

Comme 5 : emplois «plus pragmatiques (...) tels : la valeur qualifiante, métaénonciative et adverbiale »;

Comme 6 : comme dans la locution conjonctive à valeur concessive comme que;

et en quatre catégories pour comment:

Comment 1 : interrogation et exclamation directe et indirecte ;

Comment 2 : valeur analogique, qualifiante et adverbiale ;

Comment 3: expression de la conséquence, de la finalité, du temps et de la cause ;

Comment 4 : locution conjonctive concessive comment que.

L'évolution de chacune de ces valeurs est ensuite analysée en détail, par période et en confrontant les analyses modernes aux conceptions contemporaines. Elle prend ensuite en compte les phénomènes de concurrence qui se manifestent entre les deux termes, lesquels permettent également d'éclairer leurs évolutions respectives.

Retrouvant alors la question de la grammaticalisation, V. Wielemans s'appuie sur les analyses de M. Pierrard pour montrer la façon dont se réorganise le «système de $\operatorname{com}(e) »$, autrement dit les relations entre ses différentes valeurs d'emploi. À l'origine, comme exprimait ainsi « la coïncidence modale entre propositions », permettant l'expression de divers degrés d'identité autant que d'une valeur temporelle. Par la suite, en raison de phénomènes de concurrence dans l'expression de l'identité, comme en vient à n'exprimer plus qu'une « conformité « indéfinie » entre comparandes » (2005: \3.5), d'où peuvent être dérivées les valeurs contemporaines.

Enfin, la dernière recherche à s'inscrire dans une visée synthétique est celle menée par M. Pierrard au travers de différents articles (1998, 1999 \& 2001). Si le premier et le dernier s'attachent à des sous-ensembles de valeurs plus qu'ils ne se consacrent à l'entier des emplois, mis en parallèle avec le second, ils en proposent une analyse globale principalement axée sur l'évolution de comme.

Le premier de ces trois articles (1998) est ainsi consacré au comme "relatif à antécédent» en ancien français et se concentre sur «les tours (...) où comme semble fonctionner comme forme de reprise d'une source adverbiale» (1998: 127) et sur la grammaticalisation de la proforme indéfinie qui en est à l'origine. M. Pierrard distingue 
ainsi « trois types de constructions » dans lesquels comme est, au sein de la construction analytique, "relatif de manière et d'analogie », «relatif anaphorique » et «relatif de temps » (1998 : 129-130). La spécificité est, dans tous les cas, la «formalisation de la hiérarchisation par coïncidence au moyen du marquage des deux places saturées dans la principale et la subordonnée » (1998: 131). Il marque ainsi « la coïncidence modale entre propositions» (1998: 131-132), laquelle inclut leur identité faible (analogie) ou forte (égalité). Ce recours aux «articulants analytiques ${ }^{21}$ avec comme en second élément témoignerait ainsi d'une avancée relative dans la grammaticalisation du morphème indéfini, et, par conséquent, les changements affectant l'élément source auront également "une influence déterminante sur la réorganisation des emplois de comme » (1998: 136). Le passage du fonctionnel au pragmatique dans le cas des éléments sources entraînant le recours à des formes plus marquées et l'élimination de la valeur de conformité autant que le caractère progressivement redondant de comme dans l'expression de l'égalité contribueront à l'usage de formes concurrentes à second élément que. En revanche, une tendance aux formes synthétiques favorisera l'usage de comme pour l'expression de la comparaison analogique et, plus généralement, d'une conformité indéfinie. C'est l'ensemble du système des proformes indéfinies qui s'en trouvera ainsi réorganisé.

Dans le second article (1999), M. Pierrard s'interroge plus spécifiquement sur les

facteurs contextuels [qui] modulent les propriétés de base du morphème et l'amènent à remplir des fonctions grammaticales fort éloignées de ses attributions initiales. [PIERRARD 1999 : 133]

Il montre ainsi que

l'extension des fonctions spécifiques de comme par sa grammaticalisation en tant que connecteurs de prédicats tirera pleinement parti des propriétés de base de la P[roforme] I[ndéfinie] : un sémantisme de modalité non spécifié (...) et une mise en rapport de deux prédicats au moyen du marquage d'une coïncidence de modalité. [PIERRARD 1999 : 133]

Aussi bien comme étend-il d'abord ses emplois en tant que connecteur indiquant un niveau indéfini de conformité, notamment sous l'influence des relations entre les éléments comparés et de la nature propre de ceux-ci. L'examen cumulé de ces éléments permet alors de faire apparaitre la cohérence du fonctionnement de comme au travers de la diversité de ses emplois.

Enfin, plus résolument diachronique que le précédent, le dernier article de M. Pierrard (2001) entend répondre au double objectif

d'examiner les caractéristiques des glissements fonctionnels et sémantiques du marqueur afin de déterminer de quoi son agencement actuel est le produit

et d'illustrer

\footnotetext{
21 Le terme est repris de Jonas (1971) dont M. Pierrard s'inspire, en inscrivant ses analyses dans le cadre des théories de la grammaticalisation.
} 
la complexité du processus de grammaticalisation en soulignant l'interaction constante entre l'évolution diachronique et l'organisation synchronique, entre les propriétés du morphème et son fonctionnement dans divers contextes. [PIERRARD $2001: 294]$

Se fondant sur certains acquis de la typologie, et notamment les trois paramètres que sont la hiérarchisation, l'autonomie des prédications en jeu ainsi que la formalisation $\mathrm{du}$ marqueur assurant cette relation, il montre le cheminement suivi par comme, conduisant celui-ci d'un fonctionnement en tant que « forme de reprise d'un adverbe » au « repositionnement de ses emplois en fonctionnement absolu » (ibid.). Celui-ci s'est trouvé favorisé par la grammaticalisation parallèle du marqueur analytique, corollaire de la substitution d'un marquage fonctionnel par un marquage grammatical qui conduira à la généralisation de que et à l'abandon de comme en tant que second membre de ces marqueurs. Cela va entraîner la restructuration des emplois de comme, notamment en raison du déploiement des constructions en Préd1 comme Préd2, et produire finalement l'ensemble complexe que forment ses emplois actuels.

\section{Ouvertures}

Du parcours de l'ensemble de ces études, il est possible de tirer plusieurs enseignements, aussi bien sur l'évolution globale des emplois de comme au cours de l'histoire du français que sur les phénomènes ayant suscité un plus grand intérêt chez les linguistes. Ainsi l'intérêt porté à la filiation et à l'organisation conceptuelle des différentes valeurs sémantiques de comme se manifeste-t-il dans la plupart des travaux évoqués et assure-t-il, en quelque sorte en filigrane, un dénominateur commun qu'ont renforcé l'émergence du paradigme de la grammaticalisation et les recherches sur les proformes indéfinies

On notera cependant qu'aussi bien certaines périodes (les périodes préclassique et classique, notamment) que certains emplois (paradoxalement, les emplois les plus traditionnels de marqueur interrogatif et exclamatif) demeurent quelque peu laissés dans l'ombre. De la même façon, les relations de comme avec ses divers composés morphologiques (comment, combien), souffrent encore d'un nombre trop restreint d'études, tout particulièrement dans une perspective diachronique. 


\section{RÉFÉRENCES BIBLIOGRAPHIQUES}

BAt-Zeev ShyldKrot, H. (1989), «Conjonctions et expression temporelle-causale en français », Folia Linguistica Historica, n¹0,1-2, pp 263-281.

BAT-ZEev ShYLDKROT, H. (1995), «Subordonnées circonstancielles et dépendance sémantique. Comparaison, concession, condition : grammaticalisation et sens des connecteurs ", in Danon-Boileau, L. \& Morel, M-A. (éds), La comparaison, Faits de langue n5, pp. 145-155.

Bertin, A. (1997), L'expression de la cause en ancien français, Genève, Droz.

DARDEL, R. (de) (1983), Esquisse structurale des subordonnants conjonctionnels en roman commun, Genève, Droz.

HERMAN, J. (1963), La formation du système roman des conjonctions de subordination, Akademie-Verlag, Berlin.

IMBS, P. (1956), Les propositions subordonnées temporelles en ancien français. La détermination du moment, Strasbourg, Publications de la faculté des lettres de Strasbourg, fascicule 120. (Chapitre II : «Temps et manière : 'com(e)’ et ses composés », pp. 117-171).

JONAS, P. (1971), Les systèmes comparatifs à deux termes en ancien français, Bruxelles, Éditions de l'Université de Bruxelles.

Kuyumcuyan, A. (2006), "Comme et ses valeurs: le point de vue historique (XIVe-XVIe siècles) » in Lagorgette, D., Oppermann-Marsaux, E. \& Rodriguez Somolinos, A. (éds) Enonciation et pragmatique : Approche diachronique, Langue française $\mathrm{n}^{\circ} 149$, pp. 113-126.

Kuyumcuyan, A. (2007), «De l'Autre source à l'Autre modèle du dire. De l'origine des tours modalisateurs en comme et dire du FC», Études sur le changement lintuistique en français, Actes du colloque international Diachro II, B. Combettes \& C. Marchello-Nizia (éds), Nancy, Presses Universitaires de Nancy, pp. 157-172.

Lefeuvre, F. (2003), «Comme quoi en diachronie», in Combettes, B., Marchello-Nizia, C. \& Prévost, S. (éds) La grammaticalisation en français, Verbum XXV-4, PU Nancy, Verbum XXV-4, pp. 455-468.

MenARD, P. (1997), "Remarques sur certains emplois de com(me) en ancien français», in Kleiber, G. \& Riegel, M. (éd.) Les formes du sens. Etudes de linguistique française, médiévale et générale offertes à Robert Martin pour à l'occasion de ses soixante ans, Paris, Duculot, pp. $257-$ 267.

MORINIÈRE, M. (2008a), «L'ellipse dans les constructions introduites par comme en diachronie du français », in Pitavy, J.-C. \& M. Bigot (eds), Ellipse et effacement: du schème phrastique au discours', Publications de l'Université de Saint-Etienne, pp. 67-76.

MORINIÈRE, M. (2008b), «Évolution des constructions à attribut de l'objet en français », in Fagard, B., Prévost, S., Combettes, B. \& O. Bertrand, Evolutions en français. Etude de linguistique diachronique, Berlin : Peter Lang, 2008, pp. 285-300.

PIERrard, M. (1998), «Comme « relatif à antécédent » en ancien français : grammaticalisation de la proforme indéfinie ", in Boone, A. \& Pierrard, M. (éds) Les marqueurs de biérarchie et la grammaticalisation, Travaux de linguistique, n³6, pp. 127-146. 
Mélanie Morinière \& Thomas Verjans

Pierrard, M. (1999), «Grammaticalisation et contexte : l'extension des emplois de comme », Cadiot P. \& Ibrahim A. (éds) Analyse sémantique des prépositions, Revue de sémantique et de pragmatique, $\mathrm{n}^{\circ}$ 6, pp. 133-144.

PierRARD, M. (2001), «Grammaticalisation et restructuration fonctionnelle: comme et la subordination ", in Lagorgette D. \& Larrivée P. (éds) Représentations du sens linguistique, Munich, Lincom-Europa, pp. 293-308.

Ponchon, T. (1998), «Les emplois de com(e) en français médiéval», in Leeman, D. \& Boone, A. (éds) Du percevoir au dire. Hommage à A. Joly, Paris, L'Harmattan «sémantiques », pp. 319-350.

SOUTET, O. (1992), La concession dans la phrase complexe en français, des origines au XVTe siècle, Genève, Droz « Publications romanes et françaises », n²01.

TOBBACK, E. (2003), «La grammaticalisation et les constructions à attribut de l'objet introduit par comme» in Combettes, B., Marchello-Nizia, C. \& Prévost, S. (éds) La grammaticalisation en français, Verbum XXV-4, PU Nancy, pp. 537-548.

VÄÄNÄNEN, V. (1951), "Il est venu comme ambassadeur », "il agit en soldat » et locutions analogues en latin, français, italien et espagnol, essai de syntaxe historique et comparée, AASF, B 73, 1 Helsinki: Suomalainen Tiedeakatemia.

Wielemans, V. (2005), «L'évolution de comme et comment: le témoignage des grammairiens et des dictionnaires de l'époque ", in Circulo de lingüistica aplicada a la comunicacion, $\mathrm{n}^{\circ} 22$ [en ligne]. 